\title{
Impact of the Generator Geometry on the Size of Generated Vortex Rings
}

\author{
K. GruszkA ${ }^{a}$, P. NiegodajeW ${ }^{b, *}$ And M. BęBen ${ }^{b}$ \\ ${ }^{a}$ Częstochowa University of Technology, Department of Physics, 42-201 Częstochowa, Poland \\ ${ }^{b}$ Częstochowa University of Technology, Institute of Thermal Machinery, al. Armii Krajowej \\ 21, 42-201 Częstochowa, Poland \\ Doi: $10.12693 /$ APhysPolA.138.200 \\ *e-mail: niegodajew@imc.pcz.czest.pl
}

\begin{abstract}
Vortex rings are very stable structures that can be generated when the fluid is transiently released from the nozzle. High stability of such structures allows transporting momentum for large distances. This interesting feature opened an extensive debate on that phenomenon in academic forum. In turn, a number of research works can be found on such issues as generation, motion and decay of vortex rings. Not much attention has been put, however, on examining how the geometry of the vortex ring generator may influence on properties of generated structures and this work is aimed at filling this gap.

topics: vortex ring, vortical structure, vortex generator, vortex propagation, hot-wire anemometry
\end{abstract}

\section{Introduction}

When transiently releasing the fluid from the nozzle one may expect a formation of a vortex ring (VR) at its exit. Such structures are apparent in a number of natural processes as for instance locomotion of hydromedusas [1] or during the discharge of blood into the heart left ventricle [2]. VRs have also found some engineering applications in pulsed jets [3, 4], swimming devices [5-7] and in cooling process of electronic systems $[8,9]$. The available literature offers also information on more fundamental issues as VRs formation [10, 11], evolution [12, 13], or VRs collisions with different obstacles [14-17]. No much attention is devoted, however, to the issue of how the geometry of the generator (as for instance the orifice diameter or the length of the generator tube) could possibly influence on the size of generated VRs. Such a knowledge may be useful from the practical point of view as it may allow for controlled generation of VRs of desired size. Hence, the aim of the present work is to examine the impact of different generator geometries on the size of generated VRs with the use of hot-wire anemometry (HWA) technique [18, 19]. Based on HWA velocity measurements one may easily assess the boundaries of the generated rings and consequently examine different VR generator geometries.

\section{Experimental setup and measuring procedure}

Experimental investigation was performed with the use of the test setup schematically shown in Fig. 1 and which was also used in our previous experiments on similar issues [20, 21]. In such a configuration the Gaussian signal is generated using a personal computer (1) in the Mathematica software which is then transmitted through the amplifier (2) to the loudspeaker (3) mounted on one end of the cylindrical tube (4). The motion of the membrane pushes a column of a fluid (air) towards the axial direction $x$ and generates the VR (5) at the orifice (6) outlet. The motion of the VR can be then detected and its velocity recorded with the use of HWA (7) mounted on the automatic traversing system (8) ensuring the motion of the probe in $x$ and $r$ directions. The HWA used in the experiment was equipped with the wire, which was $0.4 \mathrm{~mm}$ in length and $3 \mu \mathrm{m}$ in diameter and it was positioned vertically to the axis of the generator. HWA was calibrated using DANTEC DYNAMICS StreamLine Pro Automatic Calibrator. The tube length $L$ and its inner diameter $D_{1}$ equalled $200 \mathrm{~mm}$ and $100 \mathrm{~mm}$, respectively. Three different orifices were used during the investigation with inner diameters $D_{2}$ equalled to 33,50 , and $66 \mathrm{~mm}$. Measurements of the velocity were performed at the fixed distance $x=3 D_{2}$, measured from the orifice outlet. Note that it was assumed that generated VRs have axisymmetric shape. Therefore, a number of measurements could be reduced to obtaining a single profile for each considered orifice diameter.

HWA measurement procedure was as follows: after positioning the probe at a certain location $x$ and $r$, one hundred VRs were generated (to get statistically reproducible results) with $1 \mathrm{~s}$ interval between consecutive excitations (to avoid potential interactions between consecutively generated structures). $2 \mathrm{kHz}$ sampling frequency appeared to be 


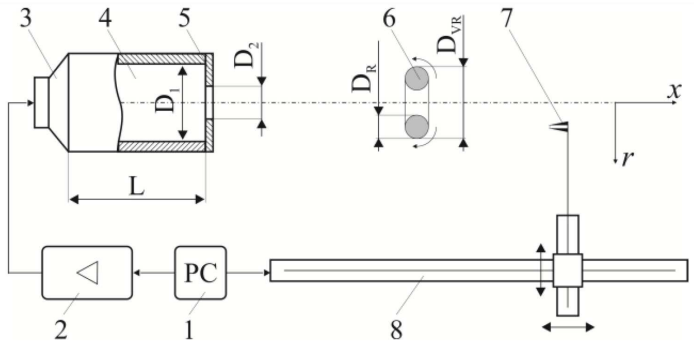

Fig. 1. Scheme of the experimental setup.

sufficient to capture all the characteristic features of the velocity signal such as local extremes. Afterwards the probe was shifted with a certain distance $\Delta r$ to made a second measurement and so on until the measured VR maximum velocity reached $5 \%$ of the highest measured one (so one could obtain the diameter $D_{\mathrm{VR}}$ of generated VRs). During the experiment the Reynolds number $\operatorname{Re}=U D_{2} / \nu$ (where $U$ is the averaged air velocity and $\nu$ is the kinematic viscosity) was kept constant at the level of $\operatorname{Re}=9600 \pm 3 \%$ at the orifice position in order to ensure identical condition at the orifice outlet for each case.

\section{Experimental results}

Figure 2 presents three superimposed consecutive velocity profiles of VRs collected at probe position $x / D_{2}=3$ and $r=0.85 D_{2}$ and for $D_{2}=33 \mathrm{~mm}$. Local extremes result from the combined progressive and rotating motion of VRs. One may observe some discrepancies between presented velocity profiles, that is why one hundred VRs were generated for each radial position analysed to get statistically representative results.

Figure 3 illustrates radial distributions of maximum $U_{\max }$ and minimum $U_{\min }$ velocities corresponding to local maximum (larger one) and local minimum, respectively (apparent in Fig. 2). Note that each point represents averaged velocity value from 100 consecutive VR shots. As can be seen, both profiles tend asymptotically to zero with increase of radial distance $r$. Therefore, either $U_{\max }$ or $U_{\min }$ can be used as a representative criterion in estimation of VR radial activity limit - in other words, in estimation of $D_{\mathrm{VR}}$ and $D_{\mathrm{R}}$ (see Fig. 1). It is interesting to note that $U_{\min }$ profile appears at certain radial distance $(r \approx 24 \mathrm{~mm})$. It is because the radial distance occupied by $U_{\text {min }}$ profile corresponds to the rotating part of VR. Moreover, the beginning of the rotating part of VR is probably related to appearance of the local minimum at $r \approx 20 \mathrm{~mm}$ in $U_{\max }$ profile, however, more data is needed to address this issue.

Figure 4 shows a direct comparison between $U_{\max }$ profiles for each considered $D_{2} / D_{1}$ ratio vs. normalised radial distance $r / D_{2}$. As results from Fig. 4, the $U_{\max }$ reaches zero at lower distances $r / D_{2}$ for higher $D_{2} / D_{1}$, i.e. for orifices with larger

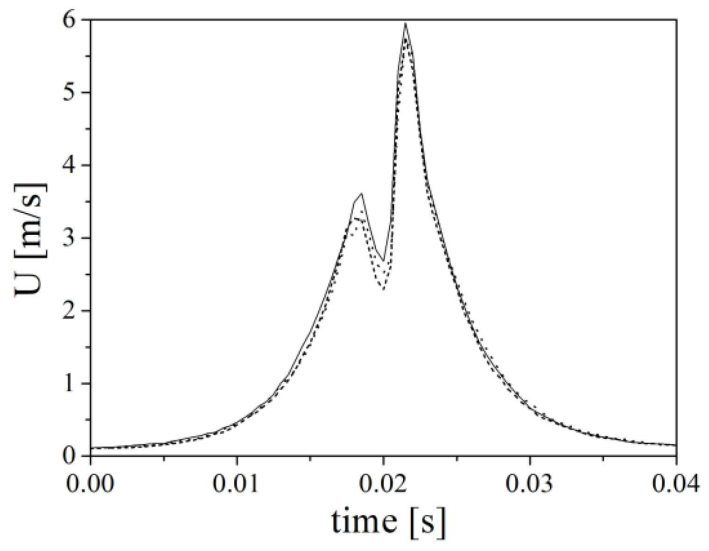

Fig. 2. Three consecutive velocity traces collected at probe position $x / D_{2}=3$ and $r=0.85 D_{2}$ and for $D_{2}=33 \mathrm{~mm}$.

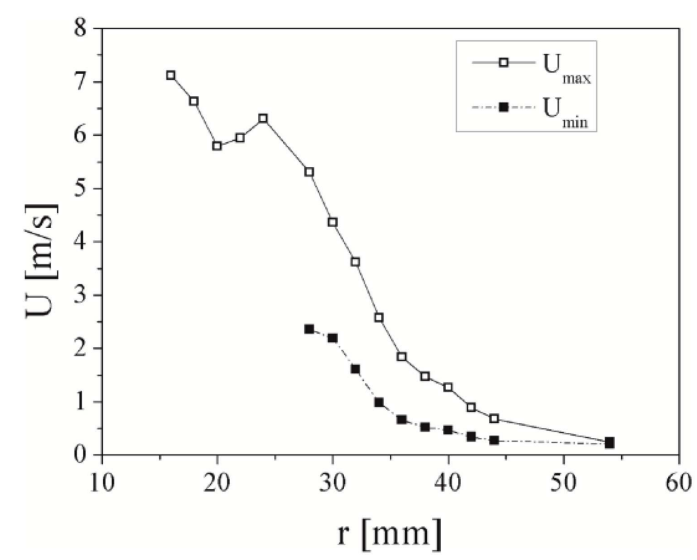

Fig. 3. Radial distributions of maximum and minimum VRs velocities.

diameters. Data from Fig. 4 can be used to estimate activity boundaries of generated vortex rings, i.e. the total diameter of $\mathrm{VR}-D_{\mathrm{VR}}-$ see Fig. 1 for the definition. One needs first to assume an identical threshold for each velocity profile. Using $5 \%$ of $U_{\max }$ threshold seems to be reasonable choice as it is an approximate error for HWA for the smallest measured velocity values. After applying such a threshold it can be estimated that the boundary VR activity (corresponding to the total ring diameter $D_{\mathrm{VR}}$ ) is located at $1.5 r / D_{2}, 1.2 r / D_{2}$ and $0.95 r / D_{2}$ for $D_{2} / D_{1}=0.33, D_{2} / D_{1}=0.5$, and $D_{2} / D_{1}=0.66$, respectively.

To get complete information about VRs geometry evolution with changing $D_{2} / D_{1}$ ratio one need to estimate the diameter of the $\mathrm{VR}$ rotating region $D_{\mathrm{R}}$ - see Fig. 1. Let us remind that diameter $D_{\mathrm{R}}$ corresponds to the radial distance occupied by $U_{\text {min }}$ profile as shown in Fig. 3. Direct comparison between $U_{\text {min }}$ profiles for each considered $D_{2} / D_{1}$ ratio as a function of normalised radial distance $r / D_{2}$ is given in Fig. 5. The results indicate identical tendency as in Fig. 4, namely the normalised diameter of the rotating region $D_{\mathrm{R}}$ decreases with increasing $D_{2} / D_{1}$ 


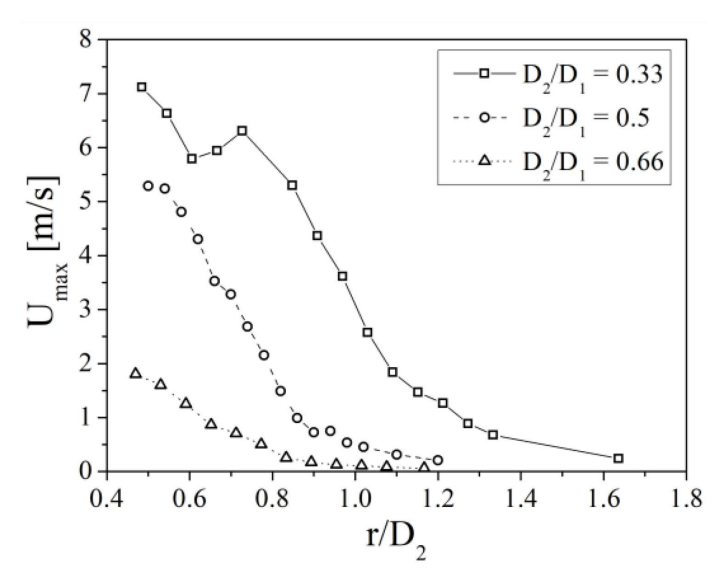

Fig. 4. Direct comparison between $U_{\max }$ profiles for each considered $D_{2} / D_{1}$ ratio as a function of normalised radial distance $r / D_{2}$.

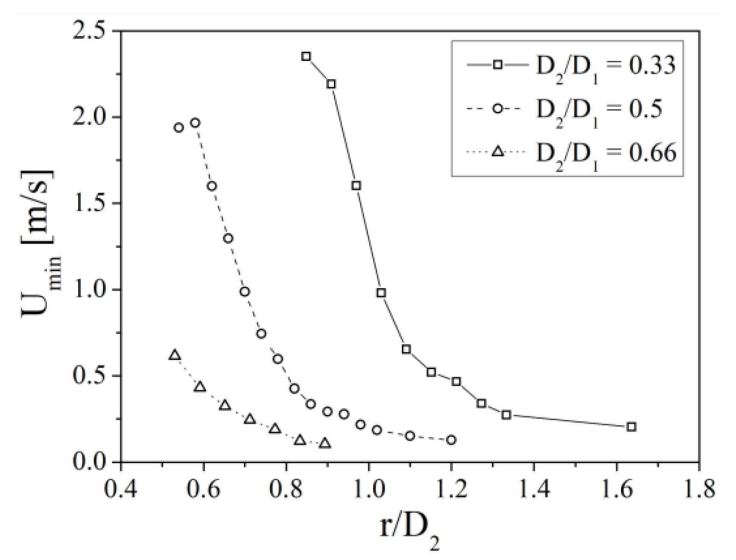

Fig. 5. Direct comparison between $U_{\text {min }}$ profiles for each considered $D_{2} / D_{1}$ ratio as a function of normalised radial distance $r / D_{2}$.

from $D_{\mathrm{R}}=0.8 r / D_{2}$ for $D_{2} / D_{1}=0.33$, through $D_{\mathrm{R}}=0.6 r / D_{2}$ for $D_{2} / D_{1}=0.5$ to $D_{\mathrm{R}}=0.4 r / D_{2}$ for $D_{2} / D_{1}=0.66$.

Taking into account the above results one may estimate the ratio between $D_{\mathrm{VR}}$ and $D_{\mathrm{R}}$ which is equal to $D_{\mathrm{VR}} / D_{\mathrm{R}}=3.75$ for $D_{2} / D_{1}=0.33, D_{\mathrm{VR}} / D_{\mathrm{R}}=4$ for $D_{2} / D_{1}=0.5$ and $D_{\mathrm{VR}} / D_{\mathrm{R}}=4.75$ for $D_{2} / D_{1}=0.66$.

\section{Conclusions}

This paper shows how the diameter of the orifice (mounted at the generator outlet) influences the size of generated VRs. Total diameter $\left(D_{\mathrm{VR}}\right)$ and diameter of the rotating part $\left(D_{\mathrm{R}}\right)$ of VRs were obtained using HWA measurements of $U_{\max }$ and $U_{\text {min }}$, respectively. The results showed that both normalised (with $r / D_{2}$ ) VR quantities vary notably when changing the orifice to tube diameter ratio $D_{2} / D_{1}$. In particular, $D_{\mathrm{VR}}$ and $D_{\mathrm{R}}$ decrease with increase of $D_{2} / D_{1}$. On the other hand, it is interesting to note that the $D_{\mathrm{VR}} / D_{\mathrm{R}}$ slightly increases with increasing $D_{2} / D_{1}$.

\section{Acknowledgments}

The present research work was funded by the National Science Centre under the Grant 2018/02/X/ST8/00924 and by the Polish National Agency for Academic Exchange under Project No. PPN/BIL/2018/1/00030.

\section{References}

[1] J.O. Dabiri, S.P. Colin, J.H. Costello, J. Exp. Biol. 209, 2025 (2006).

[2] M. Gharib, E. Rambod, A. Kheradvar, D.J. Sahn, J.O. Dabiri, PNAS 103, 6305 (2006).

[3] K. Wawrzak, A.B.A. Tyliszczak, Flow Turbul. Combust. 95, 437 (2015).

[4] A. Boguslawski, K. Wawrzak, A. Tyliszczak, J. Fluid Mech. 869 385 (2019).

[5] P.S. Krueger, A.A. Moslemi, J.T. Nichols, I.K. Bartol, W.J. Stewart, Adv. Sci. Technol. 58, 237 (2008).

[6] M. Krieg, K. Mohseni, IEEE Trans. Robot. 26, 542 (2010).

[7] P. Gil, J. Appl. Fluid Mech. 12, 293 (2019).

[8] D.S. Kercher, J. Lee, O. Brand, M.G. Allen, A. Glezer, IEEE Trans. Compon. Packag. Technol. 26, 359 (2003).

[9] A. Pavlova, M. Amitay, J. Heat Transf. 128, 897 (2016).

[10] K. Shariff, M. Field, Annu. Rev. Fluid Mech. 24, 235 (1992).

[11] M. Rosenfeld, E. Rambod, M. Gharib, J. Fluid Mech. 376, 297 (1998).

[12] I. Danaila, J. Hélie, Phys. Fluids 20, 73602 (2008).

[13] A. Saha, Y. Wei, X. Tang, C.K. Law, J. Fluid Mech. 875, 842 (2019).

[14] M.N. Musta, Measurement 88, 104 (2016).

[15] T.H. New, B. Zang, J. Fluid Mech. 833 648 (2017).

[16] T.H.N. Shengxian, S.B. Zang, Exp. Fluids 57, 109 (2016).

[17] J. Long, T.H. New, Exp. Fluids 57, 121 (2016).

[18] A. Romeos, G. Lemonis, Flow Turbul. Combust. 83, 153 (2009).

[19] M. Dziedzic, H.J. Leutheusser, Exp. Fluids 21, 315 (1996).

[20] P. Niegodajew, K. Łukasiak, H. Radomiak et al., Combust. Flame 194, 245 (2018).

[21] K. Gruszka, A. Durajski, P. Niegodajew et al., J. Phys. Conf. Ser. 1398, 12005 (2019). 